Honam Mathematical J. 34 (2012), No. 1, pp. 001-009

http://dx.doi.org/10.5831/HMJ.2012.34.1.1

\title{
ON THE EXTENDED $q$-EULER NUMBERS AND POLYNOMIALS OF HIGHER-ORDER WITH WEIGHT
}

\author{
Hyun-Mee Kim, Jongsung Choi and Taekyun Kim
}

\begin{abstract}
The purpose of this paper is to give a new construction of the extended $q$-Euler numbers and polynomials of higher-order with weight by using $p$-adic $q$-integral on $\mathbb{Z}_{p}$.
\end{abstract}

\section{Introduction}

Let $p$ be a fixed odd prime number. Throughout this paper, the symbol $\mathbb{Z}, \mathbb{Z}_{p}, \mathbb{Q}_{p}, \mathbb{C}$ and $\mathbb{C}_{p}$ will denote the ring of rational integers, the ring of $p$-adic integers, the field of rational numbers, the complex number field, and the completion of algebraic closure of $\mathbb{Q}_{p}$. The $p$-adic norm of $\mathbb{C}_{p}$ is defined by $|p|_{p}=1 / p$. We assume that $\alpha \in \mathbb{Q}$ and $\mathbb{Z}_{+}=\mathbb{N} \cup\{0\}$.

As an indeterminate, we consider that $q \in \mathbb{C}$ or $q \in \mathbb{C}_{p}$. If $q \in \mathbb{C}$, we assume that $|q|<1$. If $q \in \mathbb{C}_{p}$, the we assume thst $|1-q|_{p}<1$.

The $q$-number of $x$ is defined by $[x]_{q}=\frac{1-q^{x}}{1-q}$. Note that $\lim _{q \rightarrow 1}[x]_{q}=$ $x$.

Recently, the $q$-Euler numbers with weight $\alpha$ are defined by

$$
\tilde{\mathcal{E}}_{0, q}^{(\alpha)}=1, \quad \text { and } \quad q\left(q^{\alpha} \tilde{\mathcal{E}}_{q}^{(\alpha)}+1\right)^{n}+\tilde{\mathcal{E}}_{n, q}^{(\alpha)}=0 \text { if } n>0,
$$

with the usual convention about replacing $\left(\tilde{\mathcal{E}}_{q}^{(\alpha)}\right)^{n}$ by $\tilde{\mathcal{E}}_{n, q}^{(\alpha)}$ (see[5]).

The $q$-Euler polynomials with weight $\alpha$ also defined by

$$
\begin{aligned}
\tilde{\mathcal{E}}_{n, q}^{(\alpha)}(x) & =\sum_{l=0}^{n}\left(\begin{array}{l}
n \\
l
\end{array}\right)[x]_{q^{\alpha}}^{n} q^{\alpha l x} \tilde{\mathcal{E}}_{l, q}^{(\alpha)} \\
& =\left([x]_{q^{\alpha}}+q^{\alpha x} \tilde{\mathcal{E}}_{q}^{(\alpha)}\right)^{n}, \quad \text { for } n \geq 0 .
\end{aligned}
$$

Received September 26, 2011. Accepted October 11, 2011.

2000 Mathematics Subject Classification. 11S80, 11B68.

Key words and phrases. Bernoulli numbers and polynomials, Euler numbers and polynomials, fermionic p-adic integrlal, bosonic p-adic integral. 
Let $f \in C\left(\mathbb{Z}_{p}\right)=$ the space of continuous functions on $\mathbb{Z}_{p}$. Then the fermionic $p$-adic $q$-integral on $\mathbb{Z}_{p}$ is defined by Kim as follows ([1-16]):

$$
\begin{aligned}
I_{-q}(f) & =\int_{\mathbb{Z}_{p}} f(x) d \mu_{-q}(x) \\
& =\lim _{N \rightarrow \infty} \frac{1+q}{1+q^{p^{N}}} \sum_{x=0}^{p^{N}-1} f(x)(-q)^{x} .
\end{aligned}
$$

From (3), we have

$$
q^{n} I_{-q}\left(f_{n}\right)+(-1)^{n-1} I_{-q}(f)=[2]_{q} \sum_{l=0}^{n-1} f(l) q^{l}(-1)^{n-1-l},
$$

where $f_{n}(x)=f(x+n)(\operatorname{see}[1-16])$.

From (2) and (3), we note that

$$
\tilde{\mathcal{E}}_{n, q}^{(\alpha)}(x)=\int_{\mathbb{Z}_{p}}[y+x]_{q^{\alpha}}^{n} d \mu_{-q}(y) .
$$

Thus, by (5), we have

$$
\tilde{\mathcal{E}}_{n, q}^{(\alpha)}(x)=\frac{[2]_{q}}{(1-q)^{n}[\alpha]_{q}^{n}} \sum_{l=0}^{n}\left(\begin{array}{l}
n \\
l
\end{array}\right)(-1)^{l} q^{\alpha l x} \frac{1}{1+q^{\alpha l+1}} .
$$

Note that $\lim _{q \rightarrow 1} \tilde{\mathcal{E}}_{n, q}^{(\alpha)}(x)=E_{n}(x)$ where $E_{n}(x)$ are the $n$-th ordinary Euler polynomial which are defined by $\frac{2}{e^{t}+1} e^{x t}=\sum_{n=0}^{\infty} E_{n}(x) \frac{t^{n}}{n !}$ (see[1$16])$.

By using the fermionic multivariate $p$-adic $q$-integral on $\mathbb{Z}_{p}$, we give a new construction of the extended $q$-Euler numbers and polynomials of higher-order with weight $\alpha$.

From the extended $q$-Euler numbers and polynomials of higher-order with weight $\alpha$, we derive a new explicit formulae by those numbers and polynomials.

\section{On the extended $q$-Euler numbers of higher-order with weight $\alpha$}

In this section, we assume that $h_{1}, h_{2}, \cdots, h_{k} \in \mathbb{Z}_{+}$and $q \in \mathbb{C}_{p}$ with $|1-q|_{p}<1$. Now we consider a sequence of $p$-adic rational numbers 
as expansion of the $q$-Euler numbers and polynomials of order $k$ with weight $\alpha$ as follows:

$$
\begin{aligned}
& \tilde{\mathcal{E}}_{n, q}^{(k, \alpha)}\left(h_{1}, h_{2}, \cdots, h_{k}\right) \\
(7) & =\int_{\mathbb{Z}_{p}} \cdots \int_{\mathbb{Z}_{p}}\left[x_{1}+\cdots+x_{k}\right]_{q^{\alpha}}^{n} q^{\sum_{j=1}^{k} x_{j}\left(h_{j}-1\right)} d \mu_{-q}\left(x_{1}\right) \cdots d \mu_{-q}\left(x_{k}\right),
\end{aligned}
$$

and

$\tilde{\mathcal{E}}_{n, q}^{(k, \alpha)}\left(h_{1}, h_{2}, \cdots, h_{k} \mid x\right)$

$$
\begin{aligned}
= & \int_{\mathbb{Z}_{p}} \cdots \int_{\mathbb{Z}_{p}}\left[x+x_{1}+\cdots+x_{k}\right]_{q^{\alpha}}^{n} q^{\sum_{j=1}^{k} x_{j}\left(h_{j}-1\right)} \\
& d \mu_{-q}\left(x_{1}\right) \cdots d \mu_{-q}\left(x_{k}\right),
\end{aligned}
$$

By (7) and (8), we get

$$
\begin{aligned}
\tilde{\mathcal{E}}_{n, q}^{(k, \alpha)}\left(h_{1}, h_{2}, \cdots, h_{k}\right)= & \frac{[2]_{q}^{k}}{(1-q)^{n}[\alpha]_{q}^{n}} \sum_{l=0}^{n}\left(\begin{array}{l}
n \\
l
\end{array}\right)(-1)^{l} \\
& \frac{1}{\prod_{j=1}^{k}\left(1+q^{\alpha l+h_{j}}\right)},
\end{aligned}
$$

and

$$
\begin{aligned}
\tilde{\mathcal{E}}_{n, q}^{(k, \alpha)}\left(h_{1}, h_{2}, \cdots, h_{k} \mid x\right) & =\frac{[2]_{q}^{k}}{(1-q)^{n}[\alpha]_{q}^{n}} \sum_{l=0}^{n}\left(\begin{array}{c}
n \\
l
\end{array}\right)(-1)^{l} \frac{q^{\alpha l x}}{\prod_{j=1}^{k}\left(1+q^{\alpha l+h_{j}}\right)} \\
& =\sum_{l=0}^{n}\left(\begin{array}{l}
n \\
l
\end{array}\right)[x]_{q^{\alpha}}^{n-l} q^{\alpha l x} \tilde{\mathcal{E}}_{l, q}^{(k, \alpha)}\left(h_{1}, h_{2}, \cdots, h_{k}\right) .
\end{aligned}
$$

From (9) we note that

$$
\begin{aligned}
\sum_{l=0}^{n}\left(\begin{array}{c}
n \\
l
\end{array}\right)(-1)^{l} & \frac{q^{\alpha l x}}{\prod_{j=1}^{k}\left(1+q^{\alpha l+h_{j}}\right)} \\
= & \sum_{l=0}^{n}\left(\begin{array}{l}
n \\
l
\end{array}\right)[x]_{q^{\alpha}}^{n-l} q^{\alpha l x}(1-q)^{n-l}[\alpha]_{q}^{n-l} \sum_{s=0}^{l}\left(\begin{array}{l}
l \\
s
\end{array}\right)(-1)^{s} \\
& \left(\prod_{j=1}^{k}\left(1+q^{\alpha s+h_{j}}\right)\right)^{-1} .
\end{aligned}
$$

Therefore, by (10), we obtain the following theorem. 
Theorem 1. Let $h_{1}, h_{2}, \cdots h_{k} \in \mathbb{Z}_{+}$and $k \in \mathbb{N}$. Then we have

$$
\begin{gathered}
\sum_{l=0}^{n}\left(\begin{array}{c}
n \\
l
\end{array}\right)(-1)^{l} q^{\alpha l x}\left(\prod_{j=1}^{k}\left(1+q^{\alpha l+h_{j}}\right)\right)^{-1} \\
=\sum_{l=0}^{n}\left(\begin{array}{c}
n \\
l
\end{array}\right)[x]_{q^{\alpha}}^{n-l} q^{\alpha l x}(1-q)^{n-l}[\alpha]_{q}^{n-l} \sum_{s=0}^{l}\left(\begin{array}{l}
l \\
s
\end{array}\right)(-1)^{s}\left(\prod_{j=1}^{k}\left(1+q^{\alpha s+h_{j}}\right)\right)^{-1} .
\end{gathered}
$$

By (4), we get

$$
\begin{aligned}
& q^{h_{1}} \int_{\mathbb{Z}_{p}}\left[x_{1}+x+1\right]_{q^{\alpha}}^{n} q^{\left(h_{1}-1\right) x_{1}} d \mu_{-q}\left(x_{1}\right) \\
& =-\int_{\mathbb{Z}_{p}}\left[x_{1}+x\right]_{q^{\alpha}}^{n} q^{\left(h_{1}-1\right) x_{1}} d \mu_{-q}\left(x_{1}\right)+[2]_{q}[x]_{q^{\alpha}}^{n} .
\end{aligned}
$$

Therefore, by (11) we obtain the following theorem.

Theorem 2. For $n \in \mathbb{Z}_{+}$, we have

$$
q^{h_{1}} \tilde{\mathcal{E}}_{n, q}^{(1, \alpha)}\left(h_{1} \mid x+1\right)+\tilde{\mathcal{E}}_{n, q}^{(1, \alpha)}\left(h_{1} \mid x\right)=[2]_{q}[x]_{q^{\alpha}}^{n} .
$$

By (8) we get

$$
\begin{aligned}
& q^{\alpha x} \tilde{\mathcal{E}}_{n, q}^{(k, \alpha)}\left(h_{1}+\alpha, h_{2}+\alpha, \cdots, h_{k}+\alpha \mid x\right) \\
= & q^{\alpha x} \int_{\mathbb{Z}_{p}} \cdots \int_{\mathbb{Z}_{p}}\left[x_{1}+\cdots+x_{k}+x\right]_{q^{\alpha}}^{n} q^{\sum_{j=1}^{k} x_{j}\left(h_{j}+\alpha-1\right)} \\
& d \mu_{-q}\left(x_{1}\right) \cdots d \mu_{-q}\left(x_{k}\right) \\
= & \left(q^{\alpha}-1\right) \int_{\mathbb{Z}_{p}} \cdots \int_{\mathbb{Z}_{p}}\left[x_{1}+\cdots+x_{k}+x\right]_{q^{\alpha}}^{n+1} q^{\sum_{j=1}^{k} x_{j}\left(h_{j}-1\right)} \\
& d \mu_{-q}\left(x_{1}\right) \cdots d \mu_{-q}\left(x_{k}\right) \\
& +\int_{\mathbb{Z}_{p}} \cdots \int_{\mathbb{Z}_{p}}\left[x_{1}+\cdots+x_{k}+x\right]_{q^{\alpha}}^{n} q^{\sum_{j=1}^{k} x_{j}\left(h_{j}-1\right)} \\
& d \mu_{-q}\left(x_{1}\right) \cdots d \mu_{-q}\left(x_{k}\right) .
\end{aligned}
$$

Thus, we obtain the following theorem.

Theorem 3. For $n \in \mathbb{Z}_{+}$and $k \in \mathbb{N}$, we have

$$
q^{\alpha x} \tilde{\mathcal{E}}_{n, q}^{(k, \alpha)}\left(h_{1}+\alpha, \cdots, h_{k}+\alpha \mid x\right)
$$




$$
=\left(q^{\alpha}-1\right) \tilde{\mathcal{E}}_{n+1, q}^{(k, \alpha)}\left(h_{1}, \cdots, h_{k} \mid x\right)+\tilde{\mathcal{E}}_{n, q}^{(k, \alpha)}\left(h_{1}, \cdots, h_{k} \mid x\right) .
$$

Let $d \in \mathbb{N}$ with $d \equiv 1(\bmod 2)$. Then we have

$$
\begin{aligned}
& \tilde{\mathcal{E}}_{n, q}^{(k, \alpha)}\left(h_{1}, \cdots, h_{k} \mid x\right) \\
= & \int_{\mathbb{Z}_{p}} \cdots \int_{\mathbb{Z}_{p}}\left[x+x_{1}+\cdots+x_{k}\right]_{q^{\alpha}}^{n} q^{\sum_{j=1}^{k} x_{j}\left(h_{j}-1\right)} d \mu_{-q}\left(x_{1}\right) \cdots d \mu_{-q}\left(x_{k}\right) \\
= & \frac{[d]_{q^{\alpha}}^{n}}{[d]_{-q}^{k}} \sum_{a_{1}, \cdots, a_{k}=0}^{d-1} q^{\sum_{j=1}^{k} h_{j} a_{j}}(-1)^{\sum_{j=1}^{k} a_{j}} \\
& \int_{\mathbb{Z}_{p}} \cdots \int_{\mathbb{Z}_{p}}\left[\frac{\sum_{j=1}^{k} a_{j}+x}{d}+\sum_{j=1}^{k} x_{j}\right]_{q^{\alpha d}}^{n} \times q^{\sum_{j=1}^{k} x_{j}\left(h_{j}-1\right)} d \mu_{-q^{d}}\left(x_{1}\right) \\
& \cdots d \mu_{-q^{d}}\left(x_{k}\right) \\
(12)= & \frac{[d]_{q^{\alpha}}^{n}}{[d]_{-q}^{k}} \sum_{a_{1}, \cdots, a_{k}=0}^{d-1} q^{\sum_{j=1}^{k} h_{j} a_{j}}(-1)^{\sum_{j=1}^{k} a_{j}} \tilde{\mathcal{E}}_{n, q^{d}}^{(k, \alpha)} \\
& \left(h_{1}, \cdots, h_{k} \mid \frac{\sum_{j=1}^{k} a_{j}+x}{d}\right) .
\end{aligned}
$$

Therefore, by (12), we obtain the following theroem.

Theorem 4. For $n \in \mathbb{Z}_{+}$and $d \in \mathbb{N}$ with $d \equiv 1(\bmod 2)$, we have

$$
\begin{aligned}
& \tilde{\mathcal{E}}_{n, q}^{(k, \alpha)}\left(h_{1}, \cdots, h_{k} \mid x\right) \\
= & \frac{[d]_{q^{\alpha}}^{n}}{[d]_{-q}^{k}} \sum_{a_{1}, \cdots, a_{k}=0}^{d-1} q^{\sum_{j=1}^{k} h_{j} a_{j}}(-1)^{\sum_{j=1}^{k} a_{j}} \tilde{\mathcal{E}}_{n, q^{d}}^{(k, \alpha)}\left(h_{1}, \cdots, h_{k} \mid \frac{\sum_{j=1}^{k} a_{j}+x}{d}\right) .
\end{aligned}
$$

Let $d \in \mathbb{N}$ with $d \equiv 1(\bmod 2)$. For $N \in \mathbb{N}$, we get

$$
\begin{gathered}
X=X_{d}=\lim _{\overleftarrow{N}} \mathbb{Z} / d p^{N} \mathbb{Z}, \quad X_{1}=\mathbb{Z}_{p}, \quad X^{*}=\bigcup_{0<a<d p,(a, p)=1}\left(a+d p \mathbb{Z}_{p}\right), \\
a+d p^{N} \mathbb{Z}_{p}=\left\{x \in X \mid x \equiv a \quad\left(\bmod d p^{N}\right)\right\}
\end{gathered}
$$

where $a \in \mathbb{Z}$ lies in $0 \leq a<d p^{N}$.

Let $\chi$ be a primitive Dirichlet character with conductor $d \in \mathbb{N}$. Then we consider the generalized $q$-Bernoulli numbers of order $k$ with weight 
$\alpha$ as follows:

$$
\begin{aligned}
& \tilde{\mathcal{E}}_{n, \chi, q}^{(k, \alpha)}\left(h_{1}, \cdots, h_{k}\right) \\
= & \int_{X} \cdots \int_{X}\left(\prod_{i=1}^{k} \chi\left(x_{i}\right)\right)\left[x_{1}+\cdots+x_{k}\right]_{q^{\alpha}}^{n} q^{\sum_{i=1}^{k} x_{i}\left(h_{i}-1\right)} \\
& d \mu_{-q}\left(x_{1}\right) \cdots d \mu_{-q}\left(x_{k}\right) .
\end{aligned}
$$

By (13), we note that

$$
\begin{aligned}
& \tilde{\mathcal{E}}_{n, q}^{(k, \alpha)}\left(h_{1}, \cdots, h_{k}\right) \\
= & \frac{[d]_{q^{\alpha}}^{n}}{[d]_{-q}^{k}} \sum_{a_{1}, \cdots, a_{k}=0}^{d-1} q^{\sum_{i=1}^{k} h_{i} a_{i}}(-1)^{\sum_{j=1}^{k} a_{j}}\left(\prod_{i=1}^{k} \chi\left(a_{i}\right)\right) \tilde{\mathcal{E}}_{n, q}^{(k, \alpha)} \\
& \left(h_{1}, \cdots, h_{k} \mid \frac{\sum_{j=1}^{k} a_{j}}{d}\right) .
\end{aligned}
$$

Let $F_{q}^{\left(k, \alpha \mid h_{1}, \cdots, h_{k}\right)}(t, x)=\sum_{n=0}^{\infty} \tilde{\mathcal{E}}_{n, q}^{(k, \alpha)}\left(h_{1}, \cdots, h_{k} \mid x\right) \frac{t^{n}}{n !}$. Then, by (9), we get

$$
\begin{aligned}
& F_{q}^{\left(k, \alpha \mid h_{1}, \cdots, h_{k}\right)}(t, x) \\
= & {[2]_{q}^{k} \sum_{m_{1}, \cdots, m_{k}=0}^{\infty} q^{\sum_{j=1}^{k} h_{j} m_{j}}(-1)^{\sum_{j=1}^{k} m_{j}} \sum_{n=0}^{\infty}\left[m_{1}+\cdots+m_{k}+x\right]_{q^{\alpha}}^{n} \frac{t^{n}}{n !} } \\
(14)= & {[2]_{q}^{k} \sum_{m_{1}, \cdots, m_{k}=0}^{\infty} q^{\sum_{j=1}^{k} h_{j} m_{j}}(-1)^{\sum_{j=1}^{k} m_{j}} e^{\left[m_{1}+\cdots+m_{k}+x\right]_{q^{\alpha}} t} . }
\end{aligned}
$$

Therefore, by (14), we obtain the following theroem.

Theorem 5. Let $F_{q}^{\left(k, \alpha \mid h_{1}, \cdots, h_{k}\right)}(t, x)=\sum_{n=0}^{\infty} \tilde{\mathcal{E}}_{n, q}^{(k, \alpha)}\left(h_{1}, \cdots, h_{k} \mid x\right) \frac{t^{n}}{n !}$. Then we have

$$
F_{q}^{\left(k, \alpha \mid h_{1}, \cdots, h_{k}\right)}(t, x)=[2]_{q}^{k} \sum_{m_{1}, \cdots, m_{k}=0}^{\infty} q^{\sum_{j=1}^{k} h_{j} m_{j}}(-1)^{\sum_{j=1}^{k} m_{j}} e^{\left[m_{1}+\cdots+m_{k}+x\right]_{q^{\alpha}} t} .
$$




\section{Further Remark}

In this section, we assume that $q \in \mathbb{C}$ with $|q|<1$. As well known definition, the gamma function is defined by

(15) $\Gamma(s)=\int_{0}^{\infty} e^{-t} t^{s-1} d t, \quad$ where $\quad s \in \mathbb{C} \quad$ with $\operatorname{Re}(s)>0$.

From (15), we have

$$
\Gamma(s+1)=s \Gamma(s), \quad \text { and } \quad \Gamma(n+1)=n ! \quad(n \in \mathbb{N}) .
$$

In $\mathbb{C}$, the extended $q$-Euler polynomials of order $k$ with weight $\alpha$ are given by

$$
\begin{aligned}
F_{q}^{\left(k, \alpha \mid h_{1}, \cdots, h_{k}\right)}(t, x) & =[2]_{q}^{k} \sum_{m_{1}, \cdots, m_{k}=0}^{\infty} q^{\sum_{j=1}^{k} h_{j} m_{j}}(-1)^{\sum_{j=1}^{k} m_{j}} e^{\left[m_{1}+\cdots+m_{k}+x\right]_{q} \alpha} t \\
& =\sum_{n=0}^{\infty} \tilde{\mathcal{E}}_{n, q}^{(k, \alpha)}\left(h_{1}, \cdots, h_{k} \mid x\right) \frac{t^{n}}{n !} .
\end{aligned}
$$

For $s \in \mathbb{C}$, it is easy to show that

$$
\begin{array}{r}
\frac{1}{\Gamma(s)} \int_{0}^{1} F_{q}^{\left(k, \alpha \mid h_{1}, \cdots, h_{k}\right)}(-t, x) t^{s-1} d t \\
=[2]_{q}^{k} \sum_{m_{1}, \ldots, m_{k}=0}^{\infty} \frac{q^{\sum_{j=1}^{k} h_{j} m_{j}}(-1)^{\sum_{j=1}^{k} m_{j}}}{\left[m_{1}+\cdots+m_{k}+x\right]_{q^{\alpha}}^{s}},
\end{array}
$$

where $x \neq 0,-1,-2, \cdots$.

From (17), we can define the multiple $q$-Euler Zeta functio with weight $\alpha$ as follows: For $s \in \mathbb{C}$, define

(18) $\zeta_{q}^{(k, \alpha)}\left(h_{1}, \cdots, h_{k} \mid s, x\right)=[2]_{q}^{k} \sum_{m_{1}, \ldots, m_{k}=0}^{\infty} \frac{q^{\sum_{j=1}^{k} h_{j} m_{j}}(-1)^{\sum_{j=1}^{k} m_{j}}}{\left[m_{1}+\cdots+m_{k}+x\right]_{q^{\alpha}}^{s}}$,

where $x \neq 0,-1,-2, \cdots$

Note that $\zeta_{q}^{(k, \alpha)}\left(h_{1}, \cdots, h_{k} \mid s, x\right)$ is analytic function in whole complex $s$-plane. By using (16), (17), (18), and Laurent series, we obtain the following theroem.

Theorem 6. For $n \in \mathbb{Z}_{+}$, we have

$$
\zeta_{q}^{(k, \alpha)}\left(h_{1}, \cdots, h_{k} \mid-n, x\right)=\tilde{\mathcal{E}}_{n, q}^{(k, \alpha)}\left(h_{1}, \cdots, h_{k} \mid x\right) .
$$




\section{References}

[1] M. Can, M. Cenkci, V. Kurt, Y. Simsek, Twisted Dedekind type sums associated with Barnes' type multiple Frobenius-Euler l-functions, Adv. Stud. Contemp. Math. 18 (2009), 135-160.

[2] D. Ding, J. Yang, Some identities related to the Apostol-Euler and ApostolBernoulli polynomials, Adv. Stud. Contemp. Math. 20 (2010), 7-21.

[3] C. Chao-Ping, L. Lin, An inequality for the generalized-Euler-constant function, Adv. Stud. Contemp. Math. 17 (2008), 105-107.

[4] M. Cenkci, The p-adic generalized twisted $(h, q)$-Euler-l-function and its applications, Adv. Stud. Contemp. Math. 15 (2007), 37-47.

[5] K-W. Hwang, D.V. Dology, T. Kim, S.H. Lee, On the higher-order q-Euler numbers and polynomials with weight $\alpha$, Discrete Dynamics in Nature and Society 2011 (2011), Article ID 354329, 12pp.

[6] T. Kim, New approach to q-Euler polynomials of higher order, Russ. J. Math. Phys. 17 (2010), 218-225.

[7] T. Kim, Some identities on the q-Euler polynomials of higher order and q-Stirling numbers by the fermionic p-adic integral on $\mathbb{Z}_{p}$, Russ. J. Math. Phys. 16 (2009), 484-491.

[8] I. N. Cangul, V. Kurt,H. Ozden, Y. Simsek, On the higher-order $w-q$-Genocchi numbers, Adv. Stud. Contemp. Math. 19 (2009), 39-57.

[9] H. Ozden,I.N. Cangul, Y. Simsek, Remarks on q-Bernoulli numbers associated with their interpolation functions, Adv. Stud. Contemp. Math. 18 (2009), 41-48.

[10] Y. Simsek, Generating functions of the twisted Bernoulli numbers and polynomials associated with their interpolation functions,Adv. Stud. Contemp. Math. 16 (2008), 251-278.

[11] C.S. Ryoo, Y.H. Kim, A numerical investigation on the structure of the roots of the twisted q-Euler polynomials, Adv. Stud. Contemp. Math. 19 (2009), 131-141.

[12] C.S. Ryoo, Calculating zeros of the twisted Genocchi polynomials,Adv. Stud. Contemp. Math. 17 (2008), 147-159.

[13] C.S. Ryoo, H. Song, R.P. Agarwal, On the roots of the q-analogue of EulerBarnes' polynomials, Adv. Stud. Contemp. Math. (2004), 153-163.

[14] S.H. Rim, S.J. Lee, E.J. Moon On the q-Genocchi numbers and polynomials associated with q-Zeta function, Proc. Jangjeon Math. Soc. 12 (2009), 261-267.

[15] C. S. Ryoo, On the generalized Barnes type multiple q-Euler polynomials twisted by ramified roots of unity, Proc. Jangjeon Math. Soc. 13 (2010), 255-263.

[16] A Bayad, Modular properties of elliptic Bernoulli and Euler functions, Adv. Stud. Contemp. Math. 20 (2010), 389-401.

Hyun-Mee Kim

Division of General Education-Mathematics, Kwangwoon University, Seoul 139-701, Republic of Korea.

E-mail: kagness@khu.ac.kr 
Jongsung Choi

Division of General Education-Mathematics, Kwangwoon University, Seoul 139-701, Republic of Korea.

E-mail: jeschoi@kw.ac.kr

\section{Taekyun Kim}

Division of General Education-Mathematics, Kwangwoon University, Seoul 139-701, Republic of Korea.

E-mail: tkkim@kw.ac.kr 